\title{
COUPLING OF COMPLETELY INTEGRABLE SYSTEMS: THE PERTURBATION BUNDLE
}

\author{
B. FUCHSSTEINER \\ University of Paderborn \\ D 4790 Paderborn \\ Germany.
}

\begin{abstract}
We introduce a canonical Lie algebra in the direct sum of vector fields and 1-1-tensors, the perturbation bundle. This Lie algebra is extended to a full tensor structure and it is related to the Lie algebra obtained by coupling linear systems to nonlinear ones. Using Lie algebra isomorphisms from the original structure to the abstract perturbation bundle, new completely integrable systems are obtained. The formalism of Lax pairs is found to be a special case of the new structure.
\end{abstract}

\section{Introduction}

The starting point for this paper was the question of how a coupling between linear equations and integrable nonlinear ones has to be done such that the resulting two-component flow again is integrable. To avoid misinterpretation, we should mention that this question is nontrivial, even for ordinary differential equations. Otherwise, for example, if all such couplings were integrable then finding explicitly the eigenvectors of the Schrödinger operator would be possible for all cases where the potential fulfills an integrable ordinary differential equation; this certainly is not the case.

However, in section 4.3 we present a simple and direct method to construct such integrable couplings. The essential point, which makes the underlying construction possible, is that in the direct sum of vector fields and 1-1-tensors (perturbation bundle) we can find two different Lie algebras. One of these, the concrete one, simply results from the vector field Lie algebra of dynamical systems like

$$
\left(\begin{array}{l}
u_{t} \\
v_{t}
\end{array}\right)=\left(\begin{array}{l}
h(u) \\
\Omega(u) v
\end{array}\right)
$$

where $\Omega$ is a linear operator on the tangent bundle. The other one is an abstract algebra. not depending on any differential geometric assumptions, like affine connections which are needed for the concrete algebra. The surprising observation is that there is a one-to-one correspondence between the commuting pairs in both algebras, surprising insofar as both algebras are quite different with respect to the differential geometric set-up needed for their construction.

The correspondence between commuting pairs in both algebras opens the possibility to start from known commuting vector fields, then apply to them Lie algebra homomorphisms 
in the abstract algebra, and to interprete the resulting commuting pairs in the concrete algebra, thus obtaining commuting pairs of equations like (1.1).

Other applications of the interplay between the two algebras are the existence of embeddings of the usual Virasoro algebras for integrable systems into the perturbation bundle. There the second components are those operators which usually occur in the isospectralnonisospectral Lax pair formulations for these Virasoro algebras.

Another application, just for the amusement of the reader, is the construction of very many absolutely meaningless Lax pair formulations for arbitrary evolution equations.

In order to carry out all constructions which are needed in the paper, we first have to show that tensor structures can be built up over Lie algebra representations without asssuming that these arise from Lie modules; this is done in the first section of the paper.

\section{Tensors}

First we present the essential requirements needed to build up a tensor structure. In the subsequent construction the important point is that we do not need to start from a Lie algebra module. This, although only a slight generalization, will be needed later on when the perturbation bundle is treated.

Let a Lie algebra $\mathcal{L}$ be given, furthermore a vectorspace $\mathcal{F}$ (called scalar fields) and a representation of $\mathcal{L}$ acting on $\mathcal{F}$. That means for each $K \in \mathcal{L}$ we have a linear map $L_{K}: \mathcal{F} \rightarrow \mathcal{F}$ such that the map $K \rightarrow L_{K}$ is a Lie algebra isomorphism, i.e.

$$
L_{K_{1}} L_{K_{2}}-L_{K_{2}} L_{K_{1}}=L_{\left[K_{1}, K_{2}\right]}
$$

for all $h_{1}, K_{2} \in \mathcal{L}$.

From here we build up the tensor structure. Let $f \in \mathcal{F}$ then we denote by $\nabla f$ the lin-
ear map $\mathcal{L} \rightarrow \mathcal{F}$ given by

$$
\langle\Gamma f, K\rangle:=L_{K} f \text { for all } K \in \mathcal{L} \text {. }
$$

The map $\Gamma f$ is called the gradient of $f$. The set of gradients we dente by $\mathcal{L}^{*}$. The space $\mathcal{L}^{*}$ separates the elements of $\mathcal{L}$ since $K^{*} \rightarrow L_{K}$, being an we denote by $\mathcal{L}^{*}$. The space $F$-talued multilinear form $T$ on $(\Theta \mathcal{L})^{n} \otimes\left(\circlearrowleft \mathcal{L}^{*}\right)^{r}$, being an isomorphism, is injective. An
contravariant tensor. Observe that $n$-times covariant and $r$-times covariant and 1-times contravat the elements of $\mathcal{L}$ and $\mathcal{L}^{*}$ are embedded in the 1-times

Yow we first extend the

$$
I_{K} G:=[K, G] \text {. }
$$

For $G^{*} \in L^{*}$ we then define $L_{K} G^{*}$ by its action on the elements $G \in \mathcal{L}$

$$
\left.\left\langle L_{K} G^{*} . G\right\rangle:=L_{K} \cdot G^{*}, G\right\rangle-\left\langle G^{*}, L_{K} G\right\rangle \text {. }
$$
This we then extend to suitable maps on all tensors by defining for an arbitrary ( $n$-times
covariant and $r$-times contravariant) tensor $T$

$$
\begin{aligned}
L_{K}(T)\left(G_{1}, \ldots, G_{n}, G_{1}^{*}, \ldots, G_{r}^{*}\right):= & L_{K}\left(T\left(G_{1}, \ldots, G_{n}, G_{1}^{*}, \ldots, G_{r}^{*}\right)\right) \\
& -\sum_{i=1}^{r} T\left(G_{1}, \ldots, G_{n}, G_{1}^{*}, \ldots, L_{K} G_{i}^{*}, \ldots, G_{r}^{*}\right) \\
& -\sum_{j=1}^{n} T\left(G_{1}, \ldots, L_{K} G_{j}, \ldots, G_{n}, G_{1}^{*}, \ldots, G_{r}^{*}\right)
\end{aligned}
$$


where $G_{1}^{*}, \ldots, G_{r}^{*}$ and $G_{1}, \ldots, G_{n}$ are arbitrary elements of $\mathcal{L}^{*}$ and $\mathcal{L}$, respectively. This extension fulfills

$$
L_{K_{1}} L_{K_{2}}-L_{K_{2}} L_{K_{1}}=L_{\left[K_{1}, K_{2}\right]}
$$

$L_{K}$ is said to be the Lie derivative with respect to $K$. Purely covariant tensors are called forms ( $n$-forms if $n$-times covariant). The elements of $\mathcal{F}$ are called zero-forms.

Let $\alpha$ be some $n$-form and $K \in \mathcal{L}$, then by $\alpha \bullet K$ we denote the form where $K$ is inserted as first entry into $\alpha$. If $\alpha$ is a zero-form then we use the convention $\alpha \bullet K:=0$. Now, we define an exterior derivative $d$ on forms by

$$
d(0)=0
$$

and for arbitrary $n$-forms $\alpha$ recursively by

$$
(d \alpha) \bullet K:=L_{K}(\alpha)-d(\alpha \bullet K) \text { for all } K \in \mathcal{L} \text {.. }
$$

This derivative $d$ maps $n$-forms into $(n+1)$-forms. On the elements of $\mathcal{F}$ it coincides with the operation of taking the gradient. $d$ commutes with any Lie derivative and we have

$$
d \cdot d=0
$$

A form $\alpha$ is said to be closed if $d \alpha=0$. Gradients are closed one-forms because of (2.9).

A tensor $T$ is said to be $K$-invariant if $L_{K}(T)=0$. Observe that, by $(2.8)$ a closed covariant tensor $\alpha$ is $K$-invariant if and only if $\alpha \bullet K$ is again closed.

Sometimes it is useful not to consider all possible tensors, but rather a suitable substructure. Therefore, any substructure of the set of all tensors

(i) containing $\mathcal{F}, \mathcal{L}$ and $\mathcal{L}^{*}$

(ii) being closed against the operations $\otimes, \bullet$ as well as against all Lie derivatives and the operations of inserting any variable from $\mathcal{L}$ and $\mathcal{L}^{*}$ into elements of this substructure (iii) and being closed against forming new tensors by taking linear sums of tensors of equal
type or by interchanging entries of equal type is called a tensor structure over $\mathcal{L}$ and $\mathcal{F}$.

For the following considerations we restrict our attention to a suitable tensor structure. The basics of hamiltonian mechanics are:

Consider some antisymmetric linear operator $\Theta$ from the one-forms into $\mathcal{L}$. Define for arbitrary one-forms $\gamma_{1}, \gamma_{2}$ the brackets

$$
\left\{\gamma_{1}, \gamma_{2}\right\}:=L_{\left(\Theta \gamma_{1}\right)} \gamma_{2}-L_{\left(\Theta \gamma_{2}\right)} \gamma_{1}+d\left\langle\gamma_{1}, \Theta \gamma_{2}\right\rangle
$$

Then $\{$,$\} defines a Lie algebra structure in the one-forms if and only if for all one-forms$ $\gamma_{1}, \gamma_{2}$ we have

$$
\Theta\left\{\gamma_{1}, \gamma_{2}\right\}=\left[\Theta \gamma_{1}, \Theta \gamma_{2}\right]
$$

In that case the $\{$,$\} are called Poisson brackets (with respect to \Theta$ ) and $\Theta$ is said to be an implectic operator (or Poisson operator). For the proof of this crucial fact compare [15].

OBSERVATION 2.1: (Noethers Theorem) Let $\Theta$ be an implectic operator and let $K=\Theta d f$. Then $L_{K}(\Theta \gamma)=\Theta L_{K}(\gamma)$ for all one-forms $\gamma$. In particular: $\Theta$ and $f$ areinvariant with 
respect to $K$.

Proof. Using (2.8) we can rewrite

$$
\left\{\gamma_{1}, \gamma\right\}=L_{\left(\Theta \gamma_{1}\right)} \gamma-\left(d \gamma_{1}\right) \cdot(\Theta \gamma)
$$

Now using (2.11) and (2.12) we find for $\gamma_{1}=d f$ with (2.9)

$$
\begin{aligned}
L_{K} \Theta \gamma & =[K, \Theta \gamma]=[\Theta d f, \Theta \gamma]=\Theta\{d f, \gamma\} \\
& =\Theta L_{K} \gamma-(d \cdot d f) \bullet(\Theta \gamma)=\Theta L_{K} \gamma .
\end{aligned}
$$

This also shows the invariance of $\Theta$. The invariance of $f$ is a trivial consequence of the antisymmetry of $\Theta$.

We complete this section by some additional remarks. We observe first that conveniently we can represent 1-1-tensors $T$ as linear operators $\Omega_{T}: \mathcal{L} \rightarrow \mathcal{L}$ via

$$
\left\langle G^{*}, \Omega_{T} K^{\prime}\right\rangle:=T\left(K^{*}, G^{*}\right) \text { for all } G^{*} \in \mathcal{L}^{*}, K \in \mathcal{L} .
$$

Then, in this notation, for the Lie derivative of $\Omega_{T}$ we have

$$
L_{K}\left(\Omega_{T} G\right)=\left(L_{K} \Omega_{T}\right) G+\Omega_{T} L_{K} G
$$

which yields

$$
L_{K}\left(\Omega_{T}\right)=L_{K} \circ \Omega_{T}-\Omega_{T} \circ L_{K} .
$$

This is a special case of the product rule for Lie-derivatives, which, by use of the Jacobi identity, holds in general with respect to arbitrary tensor products. As a consequence we remark that for arbitrary "scalars" $\epsilon$ the map $\exp \left(\epsilon L_{K}\right)$ is a Lie algebra homomorphism. Here, for the definition of $\exp \left(\epsilon L_{K}\right)$ we use the application of the Taylor series of the exponential function to arbitrary tensors $T$

$$
\exp \left(\epsilon L_{K}\right) T=\sum_{n=0}^{\infty} \frac{\epsilon^{n}}{n !} L_{K^{n}}^{n}(T)
$$

and we assume that $K \in \mathcal{L}$ is such that this sum converges, which, for example, is the case when the series truncates (master symmetries). Since exp $\left(\epsilon L_{K}\right)$ is a homomorphism it follows in particular for arbitrary 1-1-tensors $\Omega$ and $G \in \mathcal{L}$ that

$$
\exp \left(\epsilon L_{K}\right)(\Omega G)=\left(\exp \left(\epsilon L_{K}\right)(\Omega)\right)\left(\exp \left(\epsilon L_{K^{-}}\right) G\right)
$$

For the generation of invariant elements in $\mathcal{L}$ the notion of hereditary operator ${ }^{1}[10,11,24]$ is important. A linear $\Phi: \mathcal{L} \rightarrow \mathcal{L}$ is said to be hereditary if

$$
\Phi L_{G}(\Phi)=L_{\Phi G}(\Phi) \text { for all } G \in \mathcal{L} .
$$

The use of this notion is well-known (see [11])

THEOREM 2.2 :If a hereditary $\Phi$ is invariant w.r.t. $K$, i.e. $L_{K}(\Phi)=0$, then the Lie algebra
generated by the

$$
\begin{aligned}
& K_{n}=\Phi^{n} K, n=0,1,2, \ldots \\
& { }^{1} \text { Using the product rule one easily sees that this is equivalent to the usual definition } \\
& \Phi^{2}[A, B]+[\Phi A, \Phi B]=\Phi([\Phi A, B]+[A, \Phi B]\} \text { for all } A, B
\end{aligned}
$$


is abelian.

Homomorphisms preserve properties like hereditariness or the implectic property.

REMARK 2.3: Let $H$ be some Lie-algebra homomorphism, then if $\Phi$ is hereditary the operator $H(\Phi)$ is again hereditary. Likewise, if $\Theta$ is implectic, then $H(\Theta)$ is again implectic.

At the end of this section we would like to mention a case where dropping the asssumption that $(\mathcal{L}, \mathcal{F})$ must be a Lie module is essential, namely in nonlinear quantum mechanics (see $[16]$ for details). Another case is presented in the forthcoming sections.

\section{Perturbation tensor bundle}

Consider a tensor structure over the Lie algebra $\mathcal{L}$ with scalars $\mathcal{F}$. Denote by $\mathcal{T}_{(n, m)}$ the set of its $n$-times covariant and $m$-times contravariant tensors. Again, as before, the elements $\Omega$ of $\mathcal{T}_{(1,1)}$ are considered as linear operators $\Omega: \mathcal{L} \rightarrow \mathcal{L}$. Observe that on the corresponding operator algebra for any Lie derivative $L_{K}$ the product rule (with respect to operator multiplication) does hold. We introduce direct sums

$$
\hat{\mathcal{L}}:=\mathcal{L} \oplus T_{(1,1)}, \quad \hat{\mathcal{F}}:=\mathcal{F} \oplus \mathcal{T}_{(1,0)} .
$$

In $\hat{\mathcal{L}}$ we define a bracket by

$$
\left[\left(K_{1}, \Omega_{1}\right),\left(K_{2}, \Omega_{2}\right)\right]:=\left(\left[K_{1}, K_{2}\right], L_{K_{1}}\left(\Omega_{2}\right)-L_{K_{2}}\left(\Omega_{1}\right)+\Omega_{1} \Omega_{2}-\Omega_{2} \Omega_{1}\right)
$$

for $K_{1}, K_{2} \in \mathcal{L}$ and $\Omega_{1}, \Omega_{2} \in \mathcal{T}_{(1,1)}$. Observe that from $\hat{\mathcal{L}}$ we have a one-to-one correspondence

$$
(K, \Omega) \rightarrow L_{K}+\Omega
$$

into (nonlocal) linear operators on $\mathcal{L}$ and that by this correspondence the second component of the bracket (3.2) goes over into the bracket given by operator commutation. The reason that this is one-to-one comes from the fact that, with respect to the manifold variable, $\Omega$ is a local linear operator on $\mathcal{L}$, whereas $L_{K}$ is nonlocal; and splitting up linear operators on $\mathcal{L}$ into local and nonlocal parts is a unique operation. Hence, (3.2) must define a lie product.

On $\hat{\mathcal{F}}$ we define a representation of this Lie product by:

$$
\hat{L}_{(K, \Omega)}(f, \gamma):=\left(L_{K}(f), L_{K}(\gamma)-\gamma \circ \Omega\right)
$$

where $K \in \mathcal{L}, f \in \mathcal{F}, \Omega \in \mathcal{T}_{(1,1)}$ and $\gamma \in \mathcal{T}_{(1,0)}$. To see that this indeed is a representation of the Lie algebra structure we compute

$$
\begin{aligned}
\hat{L}_{\left(K_{1}, \Omega_{1}\right)} \hat{L}_{\left(K_{2}, \Omega_{2}\right)}(f, \gamma)= & \left(L_{K_{1}} L_{K_{2}}(f), L_{K_{1}} L_{K_{2}}(\gamma)-L_{K_{1}}\left(\gamma \circ \Omega_{2}\right)\right. \\
& \left.+\gamma \circ \Omega_{2} \circ \Omega_{1}-L_{K_{2}}(\gamma) \circ \Omega_{1}\right) \\
= & \left(L_{K_{1}} L_{K_{2}}(f), L_{K_{1}} L_{K_{2}}(\gamma)-L_{K_{1}}(\gamma) \circ \Omega_{2}\right. \\
& \left.-L_{K_{2}}(\gamma) \circ \Omega_{1}-\gamma \circ L_{K_{1}}\left(\Omega_{2}\right)+\gamma \circ \Omega_{2} \cdot \Omega_{1}\right) .
\end{aligned}
$$

Hence we have

$$
\begin{aligned}
& \left(\hat{L}_{\left(K_{1}, \Omega_{1}\right)} \hat{L}_{\left(K_{2}, \Omega_{2}\right)}-\hat{L}_{\left(K_{2}, \Omega_{2}\right)} \hat{L}_{\left(K_{1}, \Omega_{1}\right)}\right)(f, \gamma) \\
& \quad=\left(L_{\left[K_{1}, K_{2}\right]}(f), L_{\left[K_{1}, K_{2}\right]}(\gamma)-\gamma \circ\left\{L_{K_{1}}\left(\Omega_{2}\right)-L_{K_{2}}\left(\Omega_{1}\right)+\Omega_{1} \Omega_{2}-\Omega_{2} \Omega_{1}\right\}\right) \\
& \quad=\hat{L}_{\left[\left(K_{1}, \Omega_{1}\right),\left(K_{2}, \Omega_{2}\right)\right]}(f, \gamma)
\end{aligned}
$$


which shows that $\hat{L}$ defines a Lie-derivative with respect to the Lie algebra structure in $\hat{\mathcal{L}}$.

If now, in the considerations of section 2 , we replace $(\mathcal{L}, \mathcal{F})$ by $(\hat{\mathcal{L}}, \hat{\mathcal{F}})$, then all assumptions which were needed to build up tensor calculus are fulfilled, hence we can build up a suitable tensor structure based on $(\hat{\mathcal{L}}, \hat{\mathcal{F}})$. The corresponding tensor bundle is called the pertubation bundle. In this bundle $\hat{\mathcal{T}}_{(n, m)}$ denotes the $n$-times covariant, $m$-times contravariant tensors.

We demonstrate that this new tensor bundle, in modification, naturally arises when pertubations of flows are considered. Take some path-connected $C^{\infty}$-manifold $\mathcal{M}$ (eventually infinite dimensional). Let an affine connection on $\mathcal{M}$ be given, and assume that this connection has vanishing torsion and curvature. Denote by $\nabla_{B}$ the covariant derivative in the direction of the vectorfield $B$. Let $\mathcal{L}$ now be the Lie algebra of $C^{\infty}$-vector fields on $\mathcal{M}$, and $\mathcal{F}$ shall correspondingly denote the $C^{\infty}$-scalar fields. Recall that vanishing torsion means that for all vector fields $A, B$ we have

$$
\nabla_{A} B-\nabla_{B} A=[A, B]
$$

The curvature is defined by ( $A, B$ arbitrary vector fields)

$$
R(A, B):=\nabla_{A} \nabla_{B}-\nabla_{B} \nabla_{A}-\nabla_{[A, B]} .
$$

Since curvature is assumed to vanish, parallel transport of tensors around closed loops leaves them unchanged (see [23]), hence parallel transport from one point of the manifold to another does not depend on the path taken.

Denote by $u$ the manifold variable. If $v$ is a tangent vector at $u_{0} \in \mathcal{M}$ then by $\Pi_{\left(u_{0}, u\right)}(v)$ we denote the tangent vector at $u$ obtained by parallel transport of $v$ from $u_{0}$ to $u$. If no confusion can arise we denote $\Pi_{\left(u_{0}, u\right)}(v)$ simply by $v$. The same notation is chosen for tensors, if $T(u)$ is a tensor field then $\Pi_{\left(u_{0}, u\right)}\left(T\left(u_{0}\right)\right)$ denotes the parallel transport of $T\left(u_{0}\right)$ from $u_{0}$ to $u$. Again, we simply write $T\left(u_{0}\right)$ instead. The fields just introduced are exactly the constant tensors, i.e. those tensors having a
vanishing covariant derivative.

One should observe that the assumptions on which this geometric situation is based are not too restrictive. Locally, this situation can be established for any manifold, one only has translation in the parameter space.

Now. consider a new manifold $\dot{\mathcal{M}}$ consisting of all pairs $(u, v)$, where $u \in \mathcal{M}$ and where r is a constant vector field. On this new manifold we consider flows of the form

$$
\left(\begin{array}{l}
u_{t} \\
v_{t}
\end{array}\right)=\left(\begin{array}{c}
h(u) \\
\Omega(u) v
\end{array}\right)
$$

where $h^{\prime}(u)$ is a vector field and $\Omega(u)$ is a 1-1-tensor. These flows are to be understood as two-component systems where a second component has been coupled linearly to the nonoriginal equation $u_{t}=h(u)$.

Taking the commutator of the infinitesimal generators of these flows we obtain

$$
\left[\left(\begin{array}{c}
K_{1}(u) \\
\Omega_{1}(u) v
\end{array}\right),\left(\begin{array}{c}
K_{2}(u) \\
\Omega_{2}(u) v
\end{array}\right)\right]=\left(\begin{array}{c}
{\left[K_{1}, K_{2}\right]} \\
\left(\nabla_{K_{1}}\left(\Omega_{2}\right)-\nabla_{K_{2}}\left(\Omega_{1}\right)+\left[\Omega_{2}, \Omega_{1} \rrbracket\right) v\right.
\end{array}\right)
$$


where $\llbracket A, B \rrbracket:=A B-B A$ denotes operator commutators.

Introducing for vector fields $K$ the 1 -1-tensors $\nabla K$ defined by $(\nabla K) G:=\nabla_{G} K$ we claim that we can rewrite the right-hand side of $(3.8)$ as

$$
\left(\begin{array}{c}
{\left[K_{1}, K_{2}\right]} \\
-\llbracket L_{K_{1}}+\nabla K_{1}-\Omega_{1}, L_{K_{2}}+\nabla K_{2}-\Omega_{2} \rrbracket v
\end{array}\right)
$$

Proof of the claim. By the product rule for covariant derivatives we have

$$
\llbracket \nabla_{K}, \Omega \rrbracket=\nabla_{K}(\Omega) .
$$

From (3.5) we obtain

$$
\left(L_{K}+\nabla K\right) G=\nabla_{K} G \text {. }
$$

Hence

$$
\begin{aligned}
-\llbracket L_{K_{1}}+\nabla K_{1}-\Omega_{1}, L_{K_{2}}+\nabla K_{2}-\Omega_{2} \rrbracket v \\
=\llbracket \nabla_{K_{2}}-\Omega_{2}, \nabla_{K_{1}}-\Omega_{1} \rrbracket v \\
=\left(\llbracket \nabla_{K_{2}}, \nabla_{K_{1}} \rrbracket-\nabla_{K_{2}}\left(\Omega_{1}\right)+\nabla_{K_{1}}\left(\Omega_{2}\right)+\llbracket \Omega_{2}, \Omega_{1} \rrbracket\right) v \\
=\left(\nabla_{\left[K_{2}, K_{1}\right]}-\nabla_{K_{2}}\left(\Omega_{1}\right)+\nabla_{K_{1}}\left(\Omega_{2}\right)+\llbracket \Omega_{2}, \Omega_{1} \rrbracket\right) v
\end{aligned}
$$

Here the last identity came from vanishing curvature (3.6). Since $v$ is constant the term $\nabla_{\left[K_{1}, K_{2}\right]}$ vanishes and the right-hand side of (3.12) clearly equals the second line of the right side of (3.8).

Looking back at the definition (3.2) we find that (3.8) and the Lie algebra (3.2) are related since for $\Omega \rightarrow \nabla K-\Omega$ the second component of (3.9) is, up to a change of sign, equal to the second component of (3.2). We denote this new Lie algebra by $\dot{\mathcal{L}}^{T}$ and write its elements as

$\left.\left({ }_{\Omega}\right)\right)$

instead of $(K, \Omega)$ as they were denoted in $\hat{\mathcal{L}}$.

We may summarize now:

OBSERVATION 3.1: In $\hat{\mathcal{L}}^{T}$ we have a Lie-algebra defined by

$$
\left.\|\left[\begin{array}{c}
K_{1} \\
\Omega_{1}
\end{array}\right),\left(\begin{array}{c}
K_{2} \\
\Omega_{2}
\end{array}\right)\right]:=\left(\begin{array}{c}
{\left[K_{1}, K_{2}\right]} \\
\nabla_{K_{1}}\left(\Omega_{2}\right)-\nabla_{K_{2}}\left(\Omega_{1}\right)+\llbracket \Omega_{2}, \Omega_{1} \rrbracket
\end{array}\right) .
$$

The lie algebras in $\hat{\mathcal{L}}$ and $\hat{\mathcal{L}}^{T}$ are related in the following way: When

$$
\left.\|\left(\begin{array}{l}
K_{1} \\
\Omega_{1}
\end{array}\right),\left(\begin{array}{c}
K_{2} \\
\Omega_{2}
\end{array}\right)\right] \|=\left(\begin{array}{c}
{\left[K_{1}, K_{2}\right]} \\
\Omega
\end{array}\right)
$$

then

$$
\llbracket\left(K_{1}, \nabla K_{1}-\Omega_{1}\right),\left(K_{2}, \nabla K_{2}-\Omega_{2}\right) \rrbracket=\left(\left[K_{1}, K_{2}\right], \nabla_{\left[K_{1}, K_{2}\right]}-\Omega\right)
$$

Hence commuting pairs of $\hat{\mathcal{L}}$

$$
\left[\left(K_{1}, \Omega_{1}\right),\left(K_{2}, \Omega_{2}\right)\right]=0
$$


correspond uniquely to commuting pairs in $\hat{\mathcal{L}}^{T}$

$$
\left|\left[\left(\begin{array}{c}
K_{1} \\
\nabla K_{1}-\Omega_{1}
\end{array}\right),\left(\begin{array}{c}
K_{2} \\
\nabla K_{2}-\Omega_{2}
\end{array}\right)\right]\right|=0 .
$$

On $\hat{\mathcal{F}}^{T}$ a representation for $[, \mathbb{\|}$ is easily found:

$$
\tilde{L}_{\left(\begin{array}{l}
K \\
\Omega
\end{array}\right)}\left(\begin{array}{l}
f \\
\gamma
\end{array}\right):=\left(\begin{array}{c}
\nabla_{K}(f) \\
\nabla_{K}(\gamma)+\gamma \circ \Omega
\end{array}\right)
$$

Only the statement about the representation is not yet proved, but this is exactly the same proof as before, this time based on the fact that the connection is curvature free. Examples for well known notions where implicitly the Lie algebra structure of the perturbation bundle
is involved are easily found.

EXAMPLE 3.2:

(1) Consider in $\hat{\mathcal{L}}$ the commuting pair

$$
[(K, \nabla K+B),(0, \Lambda)]=0
$$

then by using

$$
L_{K}(\Lambda)=\nabla_{K} \Lambda+\llbracket \Lambda, \nabla K \rrbracket
$$

we see that relation $(3.20)$ is equivalent to

$$
\nabla_{K} \Lambda=\llbracket \Lambda, B \rrbracket
$$

hence to $(\Lambda, B)$ being a Lax pair for $u_{t}=K(u)$.

(2) By the same argument we see that

$$
[(\kappa, 0) \cdot(0, \Phi)]=0
$$

is equivalent to $L_{K} \Phi=0$ hence to $\Phi$ being a recursion operator for $u_{t}=K(u)$.

(3) Via observation 3.1 we see furthermore that when $(\Lambda, B)$ is a $\operatorname{Lax}$ pair then the flows

$$
\left(\begin{array}{l}
u_{t} \\
v_{t}
\end{array}\right)=\left(\begin{array}{c}
h(u) \\
-B(u) v
\end{array}\right)
$$

and

$$
\left(\begin{array}{l}
u_{t} \\
v_{t}
\end{array}\right)=\left(\begin{array}{c}
0 \\
-\Lambda(u) v
\end{array}\right)
$$

do commute. This generalizes the well known fact that when $v$ evolves according to $v_{t}=$
$-B(u) v$ then the spectral deco time. 


\section{Applications}

\subsection{LINEARIZED EQUATIONS}

Obviously the Lie-algebra $\mathcal{L}$ can be embedded isomorphically into any of the pertubation algebras in $\hat{\mathcal{L}}$. However, this fact does not mean that automatically the tensor structure based on $(\mathcal{L}, \mathcal{F})$ can be embedded isomorphically into $(\hat{\mathcal{L}}, \hat{\mathcal{F}})$.

However, in case of the existence of a torsion-free and curvature-free affine connection we give a simple proof that for the Lie product [,] such an embedding indeed can be

achieved. To see this we choose a matrix $\epsilon \neq 0$ with $\epsilon^{2}=0$ (say a two-by-two-matrix). Then as coefficients in front of elements from $\mathcal{L}$ and $\mathcal{F}$ we admit linear combinations of the unit matrix $I$ and $\epsilon$. To this new structure $\left(\mathcal{L}^{*}, \mathcal{F}^{*}\right)$ we extend the Lie-algebra structure, and hence the tensor structure, in the obvious way. Furthermore we embed $\mathcal{L}$ and $\mathcal{F}$ via $K \rightarrow I K, f \rightarrow I K$ into this new tensor structure.

So we may consider the tensor structure $(\mathcal{L}, \mathcal{F})$ as the $\epsilon$-free substructure of $\left(\mathcal{L}^{*}, \mathcal{F}^{*}\right)$. We choose now an arbitrary constant vector field $v$ in $\mathcal{L}$, and we consider the isomorphism $\exp \left(\epsilon L_{v}\right)$. Observe that because of $\epsilon^{2}=0$ all terms higher than first order cancel in the Taylor series of this exponential function. We now consider the isomorphic image of the tensor structure $(\mathcal{L}, \mathcal{F})$ under $\exp \left(\epsilon L_{v}\right)$. We claim that this is isomorphic to a tensor structure of $(\hat{\mathcal{L}}, \hat{\mathcal{F}})$ (pertubation bundle). To see this we write $\left(\begin{array}{l}A \\ B\end{array}\right)$ instead of $I A+\epsilon B$. Then we can write for $f \in \mathcal{F}$ and $K \in \mathcal{L}$

$$
\begin{aligned}
\exp \left(\epsilon L_{v}\right) K & =\left(\begin{array}{c}
K \\
\nabla K \cdot v
\end{array}\right) \\
\exp \left(\epsilon L_{v}\right) f & =\left(\begin{array}{c}
f \\
\langle\nabla f, v\rangle
\end{array}\right)
\end{aligned}
$$

and the Lie bracket coming out of that isomorphism is exactly the one considered in (3.8), which if rewritten leads to the Lie-algebra (3.14). Now, in order to identify elements of $\left(\mathcal{L}^{*}, \mathcal{F}^{*}\right)$ as elements in the tensor structure over $\hat{\mathcal{L}}^{T}$ we write

$$
\left(\begin{array}{c}
f \\
\nabla f
\end{array}\right) \text { and }\left(\begin{array}{c}
K \\
\nabla K
\end{array}\right) \text { instead of }\left(\begin{array}{c}
f \\
\langle\nabla f, v\rangle
\end{array}\right) \text { and }\left(\begin{array}{c}
K \\
\nabla K v
\end{array}\right) \text {. }
$$

Using

$$
L_{\left(\exp \left(\epsilon L_{v}\right) K\right)}\left(\exp \left(\epsilon L_{v}\right) f\right)=\exp \left(\epsilon L_{v}\right)\left(L_{K} f\right)
$$

we find that this rewritten as an element of $\hat{\mathcal{F}}$ yields

$$
\begin{aligned}
\hat{L}_{\left(\begin{array}{c}
k \\
\nabla K
\end{array}\right)}\left(\begin{array}{c}
f \\
\nabla f
\end{array}\right) & =\left(\begin{array}{c}
L_{K} f \\
\nabla\left(L_{K} f\right)
\end{array}\right)=\left(\begin{array}{c}
\nabla_{K} f \\
\nabla\left(\nabla_{K} f\right)
\end{array}\right) \\
& =\left(\begin{array}{c}
\nabla_{K} f \\
\nabla_{K}(\nabla f)+\nabla f \circ \nabla K
\end{array}\right)
\end{aligned}
$$

which is a special case of the representation given by (3.19). Using this map we have found 
OBSERVATION 4.1: All tensors which are invariant under the flow $u_{t}=K(u)$ are mapped by $\exp \left(\epsilon L_{v}\right)$ onto invariant tensors of

$$
\left(\begin{array}{l}
u_{t} \\
v_{t}
\end{array}\right)=\left(\begin{array}{c}
K(u) \\
\nabla K(u) v
\end{array}\right) .
$$

As a consequence, complete integrability of $u_{t}=K(u)$ yields complete integrability of the coupling between the original equation and its linearization. These arguments can be applied to any order of perturabtion, say $n$-th order. For this one only has to use instead of $\epsilon$ another nilpotent matrix $\epsilon$ with $\epsilon^{n-1} \neq 0$ and $\epsilon^{n}=0$. Certainly this result is not so surprising, but usually in the literature, a fair amount of computation is needed in order to prove this observation even for special cases (see for example [25] in case of the KdV).

In case $u_{t}=K(u)$ admits a recursion operator $\Phi$ then the corresponding operator for (4.6) is easily found. Application of $\exp \left(\epsilon L_{v}\right)$ yields that

$$
\hat{\Phi}=\left(\begin{array}{cc}
\Phi & \llbracket \nabla, \Phi \rrbracket \\
0 & \Phi
\end{array}\right)
$$

must be that recursion operator. Here $\llbracket \nabla, \Phi \rrbracket$ means the operator mapping each vector field $G \in \mathcal{L}$ onto the linear operator

$$
\nabla(\Phi G)-\Phi \nabla(G)
$$

In the case $\Phi$ is hereditary, then $\hat{\Phi}$ has the same property.

\subsection{LAX PAIR HIERARCHIES}

As we have seen, the recursion operator of a hierarchy of commuting flows can be understood as a new symmetry (with vanishing first component) for the canonical embedding of the hierachy into the perturbation bundle. The same viewpoint can be adopted for Lax pairs. However there, not the trivial embedding but a more sophisticated one is needed. Furthermore an affine connection is necessary since all constructions have to be carried out in $\dot{L}^{T}$.

We consider a Virasoro algebra of vector fields (i.e. an algebra of symmetries and mastersymmetries, or a hereditary algebra, see [4,12-14]). The commutation relations of such a Virasoro algebra are

$$
\begin{aligned}
& {\left[K_{n}, K_{m}\right]=0} \\
& {\left[\tau_{n}, h_{m}\right]=(m+\rho) K_{n+m}} \\
& {\left[\tau_{n}, \tau_{m}\right]=(m-n) \tau_{n+m}}
\end{aligned}
$$

where $\rho$ is a fixed number (depending on the hierachy under consideration), and $m, n$ run from either 0 or 1 to infinity. ${ }^{2}$ Let furthermore a $\operatorname{Lax}$ pair $\left(\Lambda, B_{1}\right)$, say for $u_{t}=K_{1}(u)$, be given. Then for almost all completely integrable systems a sequence of related isospectral and nonisospectral equations can be found in the literature (see $[3,5-8,18-22])$. Looking at

${ }^{2}$ There are also meaningful cases where the $m, n$ run from $-\infty$ to $+\infty$ (see [27]). 
those results, and reformulating them in the purely Lie algebraic setup of this paper, one discovers that there are sequences of operators $A_{m} . B_{n}, m, n \in \mathbf{B}$ such that

$$
\begin{aligned}
& \left|\left[\left(\begin{array}{l}
K_{n} \\
A_{n}
\end{array}\right),\left(\begin{array}{l}
0 \\
\Lambda
\end{array}\right)\right]\right|=0 \\
& \left|\left[\left(\begin{array}{l}
\tau_{n} \\
B_{n}
\end{array}\right),\left(\begin{array}{l}
0 \\
\Lambda
\end{array}\right)\right]\right|=\Lambda^{n+1} .
\end{aligned}
$$

Now, using the Jacobi identity, one discovers that, modulo parts commuting with $\Lambda$, these vector fields must fulfill in $\hat{\mathcal{L}}^{T}$

$$
\begin{aligned}
& \left|\left[\left(\begin{array}{l}
K_{n} \\
A_{n}
\end{array}\right),\left(\begin{array}{l}
K_{m} \\
A_{m}
\end{array}\right)\right]\right|=0 \\
& \mid\left[\left(\begin{array}{c}
\tau_{n} \\
B_{n}
\end{array}\right),\left(\begin{array}{l}
K_{m_{i}} \\
A_{m}
\end{array}\right)\right]=(m+\rho)\left(\begin{array}{c}
h_{n_{i}+n_{i}} \\
A_{m+n}
\end{array}\right) \\
& \left|\left[\left(\begin{array}{c}
\tau_{n} \\
B_{n}
\end{array}\right) \cdot\left(\begin{array}{c}
\tau_{m} \\
B_{m}
\end{array}\right)\right]\right|=(m-n)\left(\begin{array}{c}
\tau_{n+m i} \\
B_{n_{n}+m i}
\end{array}\right) .
\end{aligned}
$$

Implicitly, almost the same relation can be found in the important paper [20] of Wen-Xiu $\mathrm{Ma}$. Indeed, the uncertainty with respect to the parts commuting with $\Lambda$ is easily excluded by scaling arguments and thus the relations (4.14) to (4.16) are fully established. Hence we have found an extension of the original Lie algebra to a nontrivial Virasoro algebra in $\hat{\mathcal{L}}^{T}$. This then gives rise to a new integrable hierarchy, where the $\left(K_{m}, A_{m}\right)^{T}$ correspond to the action variables and the $\left(\tau_{m}, B_{m}\right)^{T}$ to the angle variables. As a consequence all flows

$$
\left(\begin{array}{l}
u_{t} \\
v_{t}
\end{array}\right)=\left(\begin{array}{c}
K_{n}(u) \\
A_{n}(u) v
\end{array}\right)
$$

do commute, a result which for the case of the $K d V$ was already observed by Degasperis [9] (using spectral methods).

One should observe that having established the Virasoro relations for this algebra, we now have at our disposal a powerful computational tool. since such a Virasoro algebra is finitely generated. Consequently we only need to kilow the elements

$$
\left(\begin{array}{l}
K_{1} \\
A_{1}
\end{array}\right),\left(\begin{array}{c}
\tau_{1} \\
B_{1}
\end{array}\right) \text { and }\left(\begin{array}{c}
\tau_{2} \\
B_{2}
\end{array}\right) \text {. }
$$

Then the others are simply computed by recursion

$$
\begin{aligned}
& (n+\rho) A_{n+1}:=\nabla_{T_{1}}\left(A_{n}\right)-\Gamma_{K_{n}}\left(B_{1}\right)+\llbracket A_{n}, B_{1} \rrbracket \\
& (n-1) B_{n+1}:=\nabla_{T_{1}}\left(B_{n}\right)-\Gamma_{r_{n}}\left(B_{1}\right)+\llbracket B_{n}, B_{1} \rrbracket .
\end{aligned}
$$

Other applications of this kind are possible. So for example, when one equation is obtained by another one via a series of Lie isomorphisms (in the vector field Lie algebra), then the corresponding Lax pairs can be transferred from the original equation by the same isomorphisms (canonically extended). For example, using the Lie isomorphism derivation of the cylindrical $\mathrm{KdV}$ from the $\mathrm{KdV}$ (see [17]) one easily obtains the Lax pair formulation for the cylindrical $\mathrm{KdV}$. 


\subsection{COUPLING}

Linearizations and isospectral pairs are examples of cases where a linear field has been coupled integrably to a nonlinear integrable evolution equation.

This can be generalized: Consider a sequence $K_{n}$ of commuting vector fields. Embed these isomorphically by

$$
K_{n} \rightarrow \hat{K}_{n}:=\left(K_{n}, 0\right)
$$

into $\hat{\mathcal{L}}$. They again do commute. Now, we take an arbitrary element in $\hat{\mathcal{L}}$ of the form

$$
H=(0, \Omega)
$$

and we apply the Lie algebra isomorphism $\exp \left(-\lambda \hat{L}_{H}\right)$ to the algebra generated by the $\hat{K}_{n}$. The result again is a commuting algebra. The interesting point about this isomorphism is that its application does not change the local part because the first component of $H$ is equal to zero. This we see from the formula

$$
\exp \left(-\lambda \hat{L}_{H}\right)(A, 0)=\left(A, \sum_{n=1}^{\infty} \frac{1}{n !} \lambda^{n}\left(-\operatorname{ad}_{\Omega}\right)^{n-1}\left(\nabla_{A}(\Omega)+\llbracket \Omega, \nabla A \rrbracket\right)\right)
$$

where

$$
\operatorname{ad}_{\Omega}(B):=\llbracket \Omega, B \rrbracket .
$$

Of course, in order to avoid convergence difficulties we should restrict our attention to those $\Omega$ such that the series in (4.23) truncates. Now we use the one-to-one correspndence between commuting pairs of $\hat{\mathcal{L}}$ and $\hat{\mathcal{L}}^{T}$ to see that all the flows

$$
\left(\begin{array}{c}
u_{t} \\
v_{t}
\end{array}\right)=\left(\begin{array}{c}
K_{n}(u) \\
\nabla K_{n}-\left(\sum_{n=1}^{\infty} \frac{1}{n !} \lambda^{n}\left(-\operatorname{ad}_{\Omega}\right)^{n-1}\left(\nabla_{K_{n}}(\Omega)+\llbracket \Omega, \nabla K_{n} \rrbracket\right)\right) v
\end{array}\right)
$$

do commute.

EXAMPLE 4.2: Take the KdV hierarchy, and take for $\Omega$ any polynomial in $u$ and $x$ (not containing derivatives of $u$ ). Then all these series do truncate. For $\Omega=u$ and for $K:=$
$u_{x x x}+6 u_{x} u$ we obtain

$$
\begin{aligned}
\sum_{n=1}^{\infty} \frac{1}{n !} \lambda^{n}\left(-\operatorname{ad}_{\Omega}\right)^{n-1}\left(\nabla_{K}(\Omega)+\llbracket \Omega, \nabla K \rrbracket\right) \\
\quad=-3 \lambda u_{x} D^{2}+\left(-3 \lambda u_{x x}-6 \lambda^{2} u_{x}^{2}\right) D-6 \lambda^{2} u_{x} u_{x x}-6 \lambda^{3} u_{x}^{3} .
\end{aligned}
$$

Hence, the flow

$$
\left(\begin{array}{l}
u_{t} \\
v_{t}
\end{array}\right)=\left(\begin{array}{c}
u_{x x x}+6 u_{x} u \\
v_{x x x}+6(u v)_{x}+3 \lambda u_{x} v_{x x}+\left(3 \lambda u_{x x}+6 \lambda^{2} u_{x}^{2}\right) v_{x}+6 \lambda^{2} u_{x} u_{x x} v+6 \lambda^{3} u_{x}^{3} v
\end{array}\right)
$$

belongs to a completely integrable hierarchy. This construction can be continued in many
different ways.

In this construction one should observe, that in order to obtain nontrivial pairs, the trick that an isomorphism in $\hat{\mathcal{L}}$ (instead of $\hat{\mathcal{L}}^{T}$ ) is used, is essential. If instead an isomorphism in
$\hat{\mathcal{L}}^{T}$ is taken, then the pairs obtained are to relate solutions of $u_{t}=K(u)$ with those for the two-component there is a unique way procedure. 


\subsection{LAX PAIRS GALORE}

In [2] the authors show that there are lots of meaningless Lax pairs. This observation for first order equations was already made in [26] when commenting on [1].

In the sequel, we show how to construct impressively looking Lax pairs, which are nevertheless meaningless.

Consider an arbitrary evolution equation

$$
u_{t}=K(u)
$$

where the vector field is supposed to depend on $u$ and arbitrary derivatives of $u$ with respect to $x$. Then for any differential operator $P=P(x, D)$ which does not depend on $u$ we obviously have

$$
\nabla_{K} P=\llbracket P, 0 \rrbracket
$$

i.e.

$$
[(K, 0),(0, P)]=0 .
$$

Take again

$$
H=(0, \Omega)
$$

where now $H$ is a multiplication operator depending on $x$ and $u$, but not on any derivatives of $u$. Then apply $\exp \left(-\hat{L}_{H}\right)$ to (4.29) to obtain a nontrivial, but nevertheless fake Lax pair. Because of

$$
\left|\left[\left(\begin{array}{c}
K \\
\nabla K-\sum_{n=1}^{\infty} \frac{1}{n !}\left(-\operatorname{ad}_{\Omega}\right)^{n-1}\left(\nabla_{K^{-}}(\Omega+\llbracket \Omega, \nabla K \rrbracket)\right.
\end{array}\right),\left(\begin{array}{c}
0 \\
\exp \left(-\operatorname{ad}_{\Omega}\right) P
\end{array}\right)\right]\right|=0
$$

the flow $u_{t}=K(u)$ must be an isospectral flow for the operator

$$
\Lambda=\exp \left(-\operatorname{ad}_{\Omega}\right) P
$$

Since now this operator really depends on the field variable $u$ it is, for general cases, far from obvious that this Lax formulation is absolutely meaningless. Carrying out the computation for the simple case $P=D^{2}$ and $\Omega=u$ we find the operator

$$
\Lambda=\exp \left(-\operatorname{ad}_{\Omega}\right) P=D^{2}+u_{x x}+2 u_{x} D+2 u_{x}^{2}
$$

looking almost like a decent Lax operator. That this really is not the case follows however from the fact that for any $K(u)$ of the above type we find a $B$ such that

$$
\nabla_{K} \Lambda=\llbracket B, \Lambda \rrbracket
$$

Therefore the question by what kind of conditions a Lax formulation is made meaningful deserves some attention. 


\section{References}

[1] F.A. Berezin and A.M. Perelomov, Commun. Math. Phys., 74 (1980) 129-140.

[2] F. Calogero and C. Nucci, J. Math. Phys., 32 (1991) $72-74$.

[3] W.L. Chan and Yu-kun Zheng, Inverse Problems, 7 (1991) 63-75.

[4] H.H. Chen and Y. Lee, in "Advances in nonlinear waves" [Ed. L. Debnath], Pitman, Boston-London-
Melbourne (1985) 233-239.

[5] Chen Dengyuan and Zhang Hangwei, J. Phys. A: Math. Gen, 24 (1991) 377-383.

16. Cheng, J. Math. Phys., 32 (1991) 157-162.

7) Y. Cheng, [Eds. G. Chaohao, L. Yishen, Tu. Guizhang], Research Reports in Nonlinear Physics, 8] Y. Cheng, Phys, Berlin-Heidelberg (1990) 12-22.

Physica, 46D (1990) 286-294.

9] A. Degasperis, "Linear evolution equations associated with isospectral evolutions of differential operators", University of Rome, preprint $\mathbf{3 4 5}$ (1983)

[10] B. Fuchssteiner, Nonlinear Analysis TMA, 3 (1979) 849-862.

(11) B. Fuchssteiner, Progr. Theor. Phys., 65 (1981) 861-876.

[13] B. Fuchssteiner and G. Oevel. Phys., 70 (1983) 1508-1522.

[14] B. Fuchssteiner, [Eds. S. Carillo and O in Mathematical Physics, 1 (1990) 415-479. Springer-Verlag, Berlin-Heidelberg-Now Ragnisco], Research reports in Physics - Nonlinear Dynamics,

[15] B. Fuchssteiner, in "Nonlinear System York (1990) pp114-122. Mathematics in Science and Engineering the Applied Sciences" [Eds. C.A. Rogers and W. F. Ames],

[16] B. Fuchssteiner, in "Groups and related T85 Academic Press, Boston (1991) pp211-256. R. Gielerak, J. Lukierski and Z Polated Topics, Praceedings of the first Max Born Symposium" [Eds.

[1i] B. Fuchssteiner, “Integrable Nonlinear , Kuwer, Dordrecht (1992) ppl65-178. preprint (1992)

[18] Ma Wen-Xiu, Science in China, 34 (1991) 769-782

[19] Ma Wen-Xiu, J. Phys. A. Math. Gen. 23 (1990) 270

[20] Ma Wen-Xiu, "Lax representation. Gen., 23 (1990) 2707-2716. archies of evolution equations" ${ }^{n}$. Inst. Max operator algebras of isospectral and nonisospectral hier-

[21] Ma Wen-Xiu, "The algebraic structures of isan University, Shanghai, preprint (1991) systems". Inst. Math. Fudan University, Shanghairal Lax operators and applications to integrable

[22] Ma Wen-Xiu, "An approach for constructing noi, preprint (1991) Math. Fudan University, Shanghai, prepring nonisospectral hierarchies of evolution equations", Inst.

[23] E. Nelson, -Tensor Analysis, Princeton Unt (1991)

[24] W. Oevel, in -Topics in Soliton Theory University Press, Princeton NJ (1967)

B. Fuchssteiner and M. Kruskal], World Scientific solvable Nonlinear equations" [Eds. M. Ablowitz,

[25] K.M. Tamizhmani and M. Laksmanan Scientific, Singapore (1987) pp108-124.

[26] G. Wilson, "On a so-called Lax equation of B. A: Math. Gen., 16 (1983) 3773-3782. unpublished note.

[2-] H. Thang. G. Tu. W. Oevel and B. Fuchssteiner, J. Math. Phys., 32 (1991) 1908-1918. 